\title{
AUTOMAÇÃO VERTICAL DE PORTOS - SOLUÇÕES MULTINÍVEL PARA TOTAL EFICIÊNCIA EM CONTROLE E INFORMAÇÃO*
}

Valter Félix Júnior Carvalho
Alex Robson do Nascimento Ricardo Bohn ${ }^{3}$

\section{Resumo}

A operação de um porto de minério é um campo fértil para boas práticas em integração de sistemas. Por tratar um produto normalmente sem alteração de características ao longo do processo, é possível analisar essa integração exclusivamente sob a ótica dos sistemas envolvidos. Dada a quantidade de sistemas independentes e complexos, o estabelecimento de interfaces se torna um fator essencial para o sucesso do projeto. Por outro lado, a necessidade de persistência de informações consolidadas, como informações de rotas e de planos de carregamento, torna interessante o uso de Sistemas de Bancos de Dados (SGBD) e sistemas legados. A integração de todos os sistemas necessários num projeto conciso com um fluxo de informações completo e eficiente também exige um gerenciamento vertical, que possa cuidar do acoplamento de todos os níveis de forma eficiente. Nesse mesmo caminho, o desenvolvimento coordenado com metodologias que permitem a interdisciplinaridade é essencial para complementar a tarefa da gestão, trazendo a preocupação com uma integração eficiente para o nível de desenvolvimento.

Palavras-chave: Integração de sistemas; Gerenciamento vertical; Automação industrial; Portos.

\section{Abstract}

The iron ore seaport operations can be an excellent green field for good practices in system integration. Since the product goes usually unchanged through the whole process, the integration can be seen through the transport systems perspective. Given the amount and complexity of those systems in a seaport, the establishment of good communication interfaces becomes an essential part of the process. Additionally, there's a need for persistency of consolidate information, like conveyor belt routes and ship loading plans, which can be addressed using database and legacy systems. The integration of all those systems in a concise and efficient information flow demands a great management effort, which needs to be vertically integrated in order to couple with all the development levels. Following these efforts, comes the agile development practices, which promotes the multidisciplinary work, making sense of all the management efforts and making the full system integration a reality.

Keywords: System integration; Seaport automation; Management; Railroads.

Analista de Sistemas, IHM Engenharia, Belo Horizonte, MG, Brasil.

Gerente de Engenharia, Diretoria de Implantação, Porto Sudeste, Iguaí, RJ, Brasil.

Coordenador de Eng. Elétrica e Automação, Jacobs Guimar, Iguaí, RJ, Brasil. 


\section{INTRODUÇÃO}

A partir dos anos 90, os sistemas de controle começaram um novo ciclo de modernização, recebendo características de sistemas abertos de programação de alto nível, tais como orientação a objetos, tags mnemônicos com independência de áreas de memória e bases de dados integradas com níveis superiores, como supervisão e PIMS.

Tal movimento permitiu uma segunda revolução na automação industrial. Se a primeira se deu com o barateamento e miniaturização dos microprocessadores, permitindo a criação de CLP's mais rápidos, baratos e com menor consumo de energia, a segunda se deu com a possibilidade de integração forte e simplificada dos sistemas de controle com os níveis superiores.

Dessa forma, com a possibilidade de reuso de programas, com o advento de blocos pré programados para realizar diversas funções e, sobretudo, com a possibilidade de unificação de bases de dados entre CLP's e sistemas superiores, a automação mudou seu paradigma, passando a integrar, definitivamente, o portfólio de sistemas de informação das empresas.

Sem perder suas características essenciais de controle, os sistemas de automação passaram a ser vistos como a origem dos dados consolidados a serem utilizados pelas áreas estratégicas e de processo das empresas. Com isso o desenho dos sistemas de automação passou a ser pensado não simplesmente como o desenho de sistemas de controle isolados, mas sim de sistemas de controle e informação, surgindo aí a preocupação com o formato dos dados, as redes e os módulos lógicos utilizados na programação, todos pautados por um pensamento sistêmico elaborado. Paralelamente, outra revolução se deu na área de gestão, visto a consolidação do conceito de PMO e utilização de metodologias ágeis, tais como Scrum. Essas implementações tornaram a interdisciplinaridade uma regra na maioria dos projetos, dando vantagem competitiva às empresas que passaram a aplicar esses modelos.

Em relação à gestão de projetos, as novas ferramentas passaram a permitir um controle mais nítido e eficiente de iniciativas multidisciplinares num mesmo projeto, permitindo, desta forma, ganhos em eficiência e transparência nas linhas base de desenvolvimento.

Em relação ao desenvolvimento técnico, o uso das metodologias ágeis, multidisciplinares em essência, permitiu treinamentos mais eficientes, dentro do próprio ambiente do projeto, a equalização de conhecimentos e competências da equipe e uma comunicação extremamente eficiente entre as diferentes áreas.

Dessa forma, o trabalho multidisciplinar se tornou não somente possível, mas desejável em projetos que envolvessem uma faixa vertical de tecnologias, por promover a facilidade de comunicação e acompanhamento, aliada à rapidez de definições e entregas.

A operação portuária de minério é uma atividade que pode se beneficiar intensamente de um projeto tecnológico vertical, por possuir um produto único, 0 minério, que é transferido entre diversos sub-sistemas, como Viradores de Vagões, Rotas de Correias, Pátios e Carregadores de Navios. O acompanhamento desse material em toda a cadeia de transporte é capaz de fornecer à coordenação do porto importantes informações sobre o estado em tempo real do sistema e previsões de estados futuros, como disponibilidade de pilhas, rotas de carregamento, etc.

Nesse trabalho, apresentamos um pequeno estudo de implantação de uma tecnologia de automação e TI para um porto genérico, considerando o transporte de minério desde os viradores de vagões até o carregamento nos navios. Ao longo da 
cadeia de transporte, analisou-se o uso de cada sistema de automação e TI, bem como suas interfaces. Enfim, fez-se também uma análise dos sistemas de TI que permeiam, paralelamente, todo o processo (PIMS e MES) e como os mesmos também podem se beneficiar de um projeto tecnológico integrado.

Por último, segue uma breve apresentação do projeto do Porto Sudeste, em Itaguaí/RJ, onde a Ihm Engenharia implementou o controle de rotas do porto, além de diversas interfaces e sistemas de $\mathrm{TI}$, bem como a coordenação de projeto para os sistemas individuais desenvolvidos por terceiros, cuidando das interfaces com os Viradores, Máquinas de Pátio, Carregadores e MES.

O objetivo final dessa apresentação é mostrar, através de uma base teórica e com um exemplo de um projeto já consolidado e em fase de implantação, como a integração de tecnologias pode tornar muito mais eficiente a implantação de um projeto tecnológico portuário greenfield, mostrando os desafios que se apresentam e quais as vantagens em relação a um projeto multi-fornecedor com baixa integração entre as áreas de desenvolvimento.

Sempre com o foco na eficiência, simplicidade e alto valor agregado, acredita-se que a verticalização das soluções, desde que a empresa fornecedora possua as competências técnicas e gerenciais apropriadas, fornece ganhos expressivos em prazos, simplicidade e efetividade do sistema.

\section{OPERAÇÃO PORTUÁRIA DE MINÉRIO}

A operação portuária de minério nos fornece um dos mais interessantes cases de integração de sistemas. Por lidar com um produto que transita, não transformado, por diversos sistemas, elimina-se a complexidade da modificação de peças com acréscimo de insumos e transformações de massa e nos permite analisar uma cadeia de processo apenas pela ótica de suas diferentes áreas.

Como exemplo nesse estudo, consideramos o início do processo como o recebimento, via ferroviária, do minério, sendo o mesmo transportado por correias para as máquinas de pátio que vão enfim criar as pilhas de estocagem. Em seguida, as máquinas recuperam esse minério, levando o mesmo por outro conjunto de correias até as máquinas de carregamento de navio, que enfim se encarregam de carregar o minério para os navios.

Durante todo esse processo, temos geração de diversas informações, referentes às rotas utilizadas pelo minério em todas as etapas, ao mapeamento de pátios, aos planos de carregamento e aos balanços de massa ao longo da cadeia de transporte. Com isso, uma automação completa e verticalizada pode ser implementada, permitindo não só o controle preciso de toda a cadeia, como a geração de informações consolidadas em todos os pontos da mesma.

Nesse estudo tratamos como a integração dos sistemas de CLP, Supervisório, Bancos de Dados, PIMS e sistemas de Nível 3 e 4 podem se combinar para fornecer um sistema eficiente e rico em informações relevantes para o transporte e embarque de minério de ferro em um porto.

\subsection{Descarregamento Ferroviário}

O minério vem através de composições ferroviárias que são direcionadas para viradores de vagões, onde são descarregados e enviados para as correias da rota de carregamento do pátio. 
Nessa etapa, considerando o transporte do minério sendo realizado pela ferrovia de outra empresa, temos um conjunto de interfaces para ser tratado, onde as informações de quantidade de minério por composição e por vagão devem ser transmitidas ao sistema, para posterior alimentação das informações sobre o minério a ser estocado nos pátios.

Essas interfaces, normalmente, utilizam de web-services ou alguma comunicação proprietária, em ambos os casos podendo fazer a troca de dados por meio de contêineres $\mathrm{xml}$.

Uma vez disponibilizados esses dados, é necessário fazer a persistência dos mesmos, para que possam, ao longo do processo, serem acessados pelo sistema de controle.

São necessárias também informações a respeito da composição para o sistema de controle, que precisa verificar o vagão sendo descarregado e a massa presente no mesmo.

Nesse panorama, temos o envolvimento dos seguintes sistemas:

- Nível 4: Sistema de transferência de informações de vagões, via web-services

- Nível 2: Sistema de supervisão

- Nível 1: Sistema de controle

- SGBD para transferência das informações do nível 4 para o nível 2.

Em relação às interfaces, temos:

- Interface entre nível 4 e Banco de Dados

- Interface entre Banco de Dados e Sistema Supervisório

- Interface entre diferentes Sistemas de Controle e Sistema Supervisório.

\subsection{Sistema de Rotas}

Uma vez descarregado e direcionado para as rotas, o minério é pesado nas balanças, usualmente redundantes e instaladas nas primeiras correias. Essa informação é então registrada no banco de dados para ser usada no cálculo do balanço de massa.

A quantidade de rotas disponíveis será tão maior quanto maior for a quantidade de correias e chutes de transferência presentes no sistema, sendo que, em alguns casos, as combinações podem chegar às centenas.

Tal número de combinações, naturalmente, não poderia ser armazenado no CLP e uma solução considerando o armazenamento de rotas em arquivos geridos pelo sistema Scada não seria a mais eficiente, uma vez que esses sistemas não são especializados em armazenar e recuperar grandes quantidades de dados.

Dentre várias soluções possíveis, se destaca o uso de um SGBD para armazenamento de todas as combinações de rotas, cadastro e modificação das mesmas. Tal sistema pode ser acoplado ao sistema Scada como interface, evitando assim a programação de interfaces dedicadas, o que tornaria mais complexo tanto o desenvolvimento quanto a operação.

Uma vez consolidada essa comunicação entre o SGBD e o Scada, o CLP pode se encarregar apenas de armazenar as rotas correntes, em operação, uma vez que nesse caso são necessárias informações de controle em tempo real, como estados e defeitos dos equipamentos e da rota como um todo.

Temos assim uma sinergia entre o SGBD, o Scada e o CLP, o que permite o cadastramento e acesso em curto tempo de todas as rotas possíveis no sistema, bem como o tradicional controle das rotas em operação. 
Nesse panorama, temos o envolvimento dos seguintes sistemas:

- SGBD para armazenamento, cadastro e recuperação das rotas

- Sistema de supervisão

- Sistema de CLP

Em relação às interfaces, temos:

- Interface entre SGBD e Sistema de Supervisão

- Interface entre sistema de supervisão e CLP

- Interface indireta entre SGBD e CLP

\subsection{Sistema de Pátios}

O minério circulando no sistema de rotas pode ser direcionado para armazenamento em pátios de estocagem ou, em alguns casos, para embarque direto através dos carregadores de navios.

No caso dos pátios, a rota selecionada termina em máquinas empilhadoras de minério, que são responsáveis por formar as pilhas.

Essa etapa é de grande importância no processo, uma vez que a pilha representa o estoque do porto, sendo necessário coletar o máximo de informações sobre a mesma e algumas das informações precisam ser consolidadas no momento do empilhamento (perfil de pilha, posição, etc.). Nesse caso, temos uma necessidade grande de integração dos sistemas de controle, supervisão, banco de dados e, em alguns casos, sistemas de visão artificial para análise e armazenamento dos perfis de pilhas.

Nessa etapa, a verificação da quantidade de material destinado à pilha pode ser feita em vários pontos, sendo usual o uso simultâneo de duas ou três soluções nesse sentido. Temos:

- Balanças nas correias de alimentação das máquinas de pátio

- Balanças nas lanças das máquinas de pátio

- Análise por visão computacional do perfil da pilha de minério

- Cálculo da massa empilhada através da medição de vazão em alguma das correias da rota

Novamente, o uso do SGBD em conjunto com o CLP e Supervisório se revela uma solução eficiente, tanto para adquirir informações das rotas destinadas ao pátio quanto para tratar as informações de pátio, pilha e posições noestoque de minério.

O sistema de cadastro de pátios, pilhas e posições é similar ao de rotas, usando o Supervisório como interface do operador, o SGBD para persistência das informações e o CLP para tratar a pilha no momento de sua alimentação ou recuperação.

\subsection{Carregadores de Navios}

Após a recuperação, o minério pode ser destinado a outro pátio ou encaminhado ao conjunto de correias que conduz ao carregador de navios. Nessa etapa, balanças podem ser instaladas nas correias do final da rota, para que a quantidade de minério transportada possa ser levantada e utilizada para o fechamento do balanço de massa.

Informações de alto nível para ajudar os sistemas subsequentes de carregamento podem ser adicionadas nesse momento e apresentadas no Scada, tais como o berço onde está o navio a ser carregado, dados do navio em carregamento e dados do porão do navio sendo carregado num determinado momento. Essas informações 
podem ser utilizadas tanto pelo equipamento carregador de navios (para saber, por exemplo, em qual porão será iniciado o carregamento, de acordo com as necessidades de arqueação) quanto pelo sistema de balanço de massa e os sistemas de nível mais alto.

Temos, nessa fase, também a interface entre CLP, SGBD e Supervisório. O banco de dados, nessa etapa, se encarrega de tratar e armazenar não só as informações de rotas, como também as informações do berço e do navio sendo carregado e a consolidação das informações do balanço de massa. Dessa forma, torna-se possível o acompanhamento completo da quantidade sendo carregada no navio, para posterior comparação com as informações de calado anotadas pelo comandante e as informações das balanças fiscais no final da linha. Dessa forma, temos as seguintes interfaces presentes nessa etapa:

- Interface entre CLP's

- Sistema Supervisório

- SGBD

- Sistemas de acompanhamento de embarque

- Sistemas de arqueação

\section{SISTEMAS DE INFORMAÇÃO}

Com o circuito de automação fechado desde o Virador de Vagões até o Carregador de Navios, temos os sistemas de informação permeando todo o processo, coletando informações para serem consolidadas para os níveis gerenciais e de engenharia de processo.

O sistema de PIMS se encarrega de capturar as informações analógicas e de funcionamento dos equipamentos, fornecendo, de forma acessível e gráfica, os estados dos pátios, das pilhas e do carregamento.

Já o MES, no nível acima, recebe tanto as informações de entrada do material no sistema, da ferrovia, quanto as informações do material ao longo de todo ele, até o carregamento. Pode receber informações tanto do SGBD quanto do PIMS.

\subsection{Interfaces}

As interfaces do PIMS, no sistema verticalizado, podem ser otimizadas para utilizar sistemas nativos, uma vez que a maioria dos fabricantes de sistemas supervisórios possuem também sistemas PIMS. Mesmo em caso de fabricantes diferentes, as interfaces OPC podem fornecer acesso transparente e simples às informações.

No caso do MES, por se tratar de um sistema geralmente dedicado a um processo específico, o mesmo depende de suas próprias interfaces. Nesse ponto a comunicação entre o projeto de automação e TI se torna realmente crítica, uma vez que a seleção das fontes de dados para o MES impactará diretamente na qualidade das informações adquiridas. Uma boa prática é concentrar todas essas informações no PIMS, criando-se uma interface para que o MES possa receber esses dados. $O$ SGBD também pode ser utilizado, sobretudo para transferir informações de escopo marginal ao sistema, como as informações de composição de vagões e de navios.

É importante manter um número reduzido de interfaces, com vistas à simplicidade de compreensão, manutenção e funcionamento do sistema. 


\section{PORTO SUDESTE}

Na implantação do projeto no Porto Sudeste, em Itaguaí/RJ, todas as boas práticas aqui apresentadas foram utilizadas, sendo a IHM responsável pelo desenvolvimento do Sistema de Rotas, incluindo aí CLP, Supervisório e Banco de Dados, Sistema de PIMS, sistema legado de acompanhamento de embarque (Kit de Bordo) e também das interfaces entre o Sistema de Rotas e os Viradores de Vagões, Máquinas de Pátio e Carregadores de Navio. A integração com MES também foi realizada pela $\mathrm{IHM}$, que se encarregou de conduzir as reuniões de alinhamento técnico e de planejamento com a empresa responsável pelo desenvolvimento.

Além disso, a IHM também cuidou da criação e aprovação de todas as interfaces com os demais sistemas envolvidos: Virador de Vagões, Máquinas de Pátio, Carregadores de Navios, bem como o webservices para receber da ferrovia informações das composições chegando no sistema.

Abaixo, apresentamos screenshots de algumas telas desenvolvidas no sistema, de forma a ilustrar a abrangência do projeto da IHM para o Porto Sudeste.

\subsection{Overview do Sistema}

Mostra toda a operação, desde o Virador de Vagões até o Carregador de Navios.

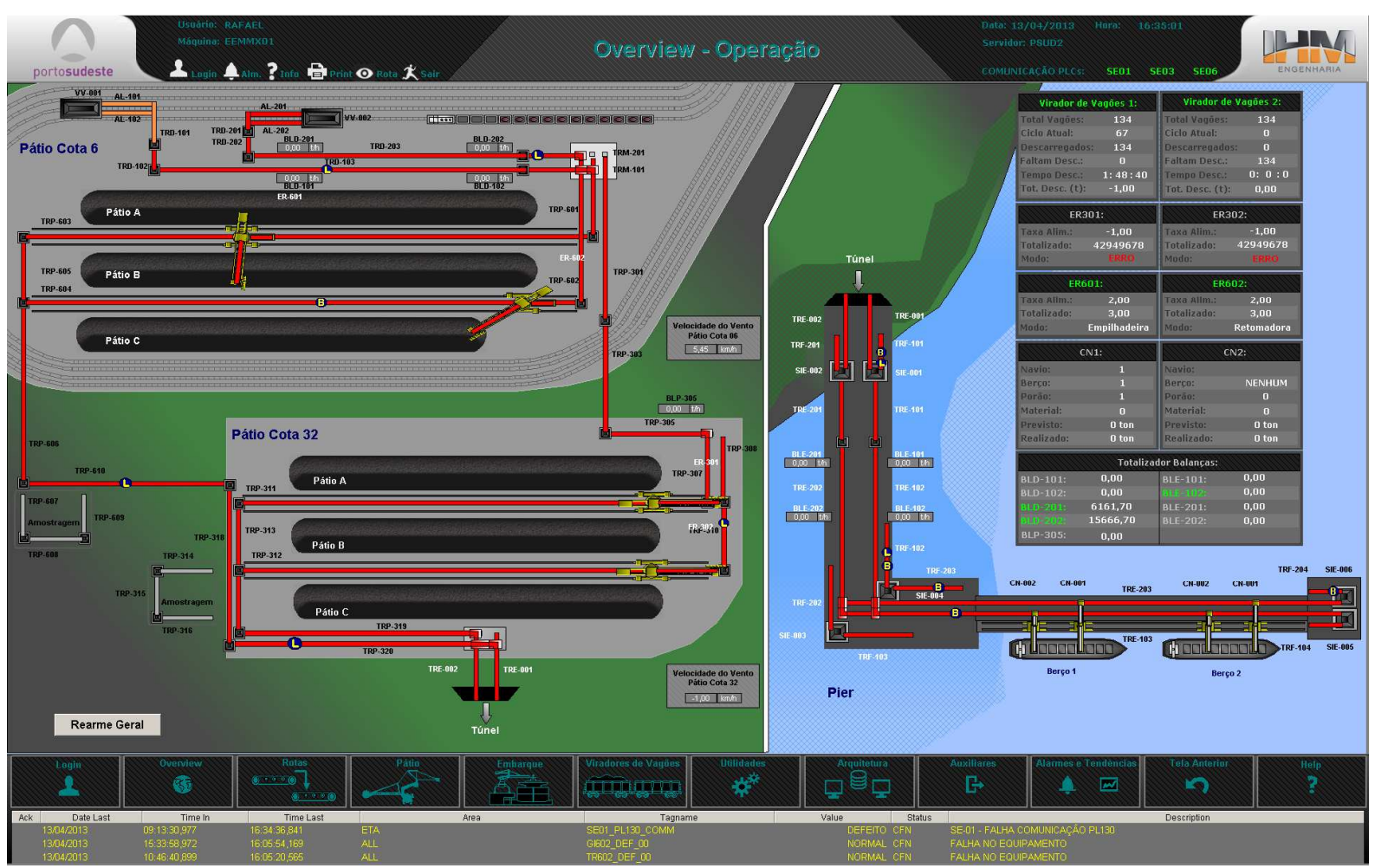

Figura 1.

\subsection{Planejamento de Descarga}

Faz o planejamento da descarga do minério dos vagões para o Sistema de Rotas. 


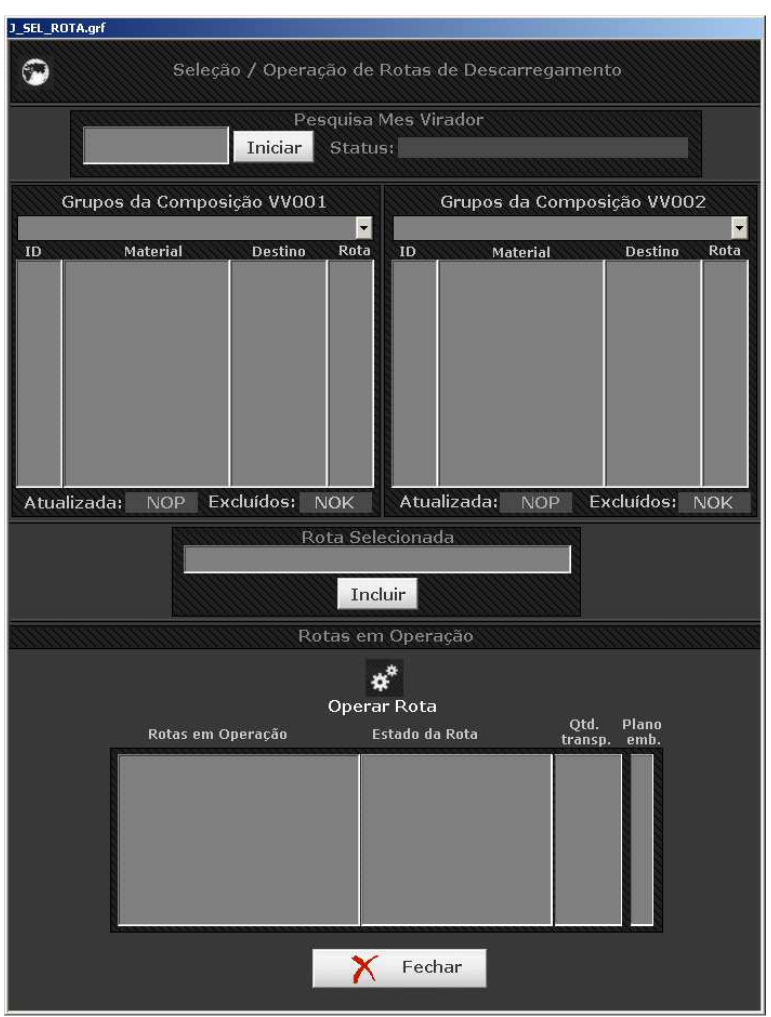

Figura 2.

\subsection{Planejamento de Embarque}

Faz o planejamento do embarque do minério, concentrando as informações do pátio com as informações do porto e as informações do MES. 


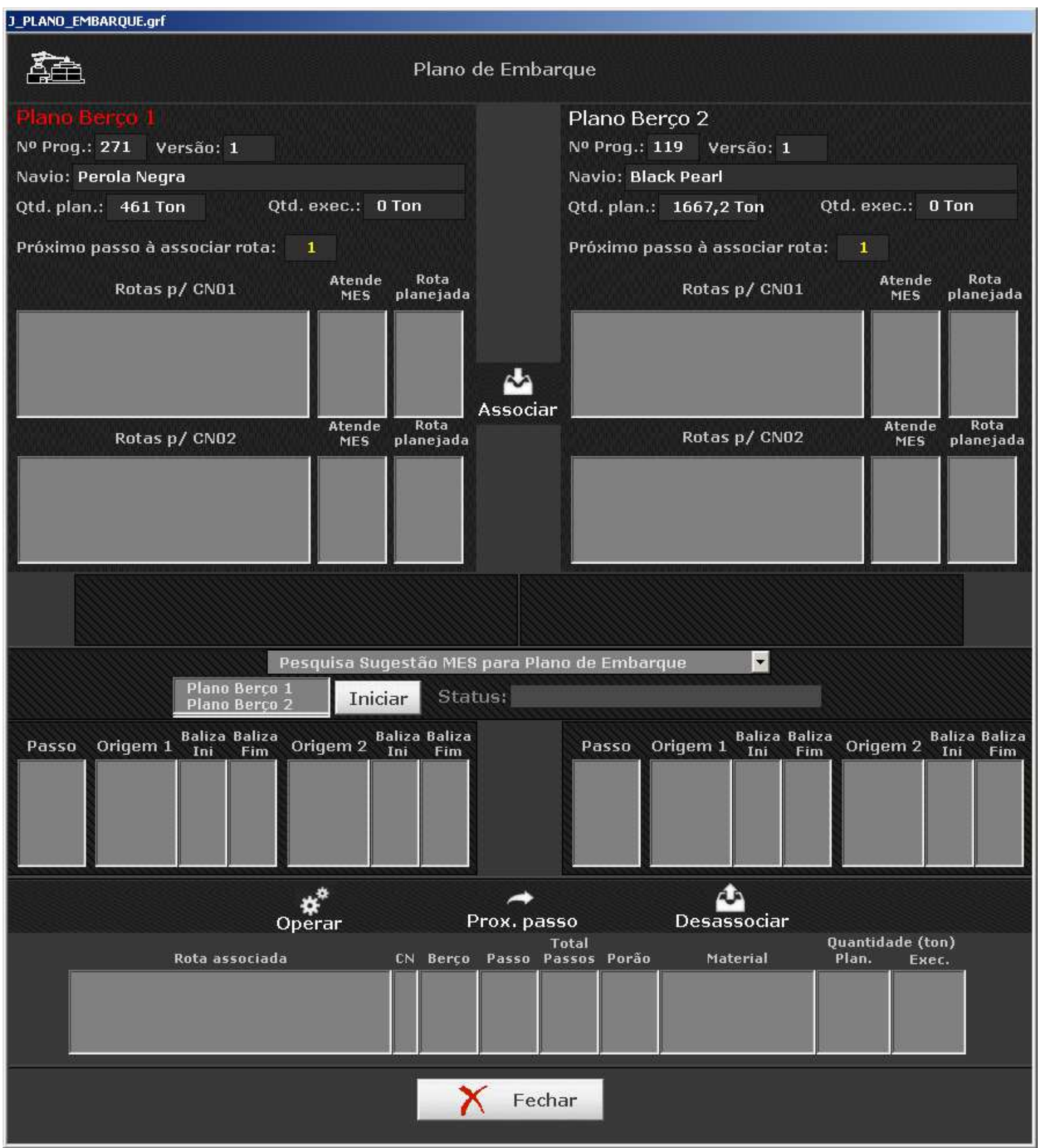

Figura 3.

\section{CONCLUSÃO}

Com um sistema de automação e de informação integrados, toda o roteamento e balanço de massa do minério no porto passa a ser controlado de forma eficiente e transparente. Neste sentido, torna-se possível para o setor de operação fazer previsões de recebimento, através das informações recebidas da ferrovia e da situação dos pátios; e também previsões de carregamento, através dos planos de embarque e sua associação com as pilhas disponíveis nos pátios.

Através das interfaces entre nível 4 e nível 2, pode-se prever o minério sendo despachado pela ferrovia, realizando planos de empilhamento para o mesmo. Uma vez passando pelos viradores de vagão, o mesmo é encaminhado paras as rotas mais adequadas. 
Através da gestão de pátios, pode-se escolher as pilhas mais adequadas para os próximos carregamentos, utilizando-se de parâmetros tais como materiais previamente estocados, proximidade física das melhores rotas de embarque, etc.

No carregamento de navios, torna-se possível escolher de antemão as pilhas que irão fornecer material para um determinado plano de carregamento, uma vez que as interfaces entre os sistemas de alto nível com os planos de carregamento e o sistema de controle permitem que as informações do plano sejam cruzadas com as informações reais de estoque nos pátios ${ }^{1}$.

$\mathrm{Na}$ fase de projeto, o gerenciamento verticalizado permite a tomada de decisões mais eficientes em relação à compra de equipamentos e de softwares, havendo a liberdade para seleção dos mesmos com base em requisitos intercomunicáveis, como por exemplo, um sistema de PIMS que possua drivers de comunicação compatíveis com o sistema SCADA e CLP.

Nessa mesma fase, a coordenação ágil do desenvolvimento, utilizando-se de metodologias como o SCRUM, tornam a execução detalhada do projeto muito mais controlável e previsível. No caso de projetos verticais, a comunicação direta entre os times de projeto e até mesmo a composição de times multidisciplinares permitem uma forte integração das tecnologias aplicadas, bem como o desenvolvimento rápido de soluções que atendam a eventuais ampliações ou modificações de escopo.

\footnotetext{
${ }^{1}$ É importante frisar que o presente estudo trata apenas do processo referente ao transporte de minério. Existem dezenas de outros sistemas em um porto, como sistemas de segurança, CFTV, sistemas fiscais, sistemas de acesso, que também podem se beneficiar de uma integração forte.
}

\section{BIBLIOGRAFIA}

1 SEGOVIA V., THEORIN A. History of Control, acessado em 13/04/2015. Disponível em: http://www.control.Ith.se/media/Education/DoctorateProgram/2012/HistoryOfControl/Van essa_Alfred_report.pdf.

2 FILHO, C.S; FONSECA, M. O. Curso Norma IEC 61131-3 Para Programação de Controladores, ISA Society Seção Belo Horizonte, 26 e 27 de Agosto de 2002.

3 Kletti, J. Manufacturing Execution Systems - MES. New York: Springer, 2007.

4 Neves, J.M.S.; Santos, F.C.A. Implantação de Tecnologias de Informação Utilizadas na Integração entre o Chão-de-Fábrica e os Sistemas ERP - XXVII Encontro Nacional de Engenharia de Produção, Foz do Iguaçu, 09 a 11 de Outubro de 2007. 